\title{
Effects of carbonated mineral water treatment in Băile Tuşnad on chronic arterial occlusive disease - a case report
}

\author{
Gabriela Dogaru' ${ }^{12}$, Ioana Stănescu ${ }^{12}$, Daniela Pop ${ }^{2}$, Marieta Motricală ${ }^{3}$, Molnár Ákos ${ }^{3}$ \\ 1. "Iuliu Haţieganu” University of Medicine and Pharmacy Cluj-Napoca, Romania \\ 2. Clinical Rehabilitation Hospital Cluj-Napoca \\ 3. Tuşnad Spa Complex - Băile Tuşnad
}

\section{ABSTRACT}

Introduction. Băile Tuşnad spa is recognized for its role in the prevention and rehabilitation treatment of cardiovascular diseases, including chronic arterial occlusive disease, due to the presence of natural therapeutic factors: carbonated mineral waters through their peripheral vasodilator effects, natural mofettes, stimulating bioclimate. Aim. The current study aimed to assess the clinical efficiency of natural therapeutic factors in Băile Tuşnad for the continuation of rehabilitation treatment in a patient with chronic arterial occlusive disease, in order to encourage walking, reduce cardiovascular risk and improve quality of life. Material and method. Patient N.M., aged 75, with multiple cardiovascular risk factors. In 2013, he was diagnosed with lower limb peripheral ischemia syndrome stage II B Fontaine, predominantly left claudication at about $100 \mathrm{~m}$, for which balloon angioplasty was performed. He attended rehabilitation treatment for 3 years in Baile Tuşnad, consisting of carbonated mineral water baths for 15 minutes, aerotherapy for 30 minutes daily for the stimulation of walking, massotherapy, kinesiotherapy, performed daily for 16 days, and in 2016, at the Rehabilitation Hospital Cluj-Napoca. He was clinically evaluated before and at the end of treatment by the Visual Analogue Scale, the 10-m walking test, adverse reactions, Doppler ultrasound. Results. At the end of treatment, an increase in the walking distance and speed, a significant improvement in the quality of gait was found; claudication occurred after $250 \mathrm{~m}$, pain in the lower limbs was improved. There were no side reactions. Conclusions. Rehabilitation treatment with natural therapeutic factors influenced the clinical and functional picture, determining a significant improvement in the quality of gait and quality of life.

Key words: chronic arterial occlusive disease, carbonated mineral waters, medical rehabilitation

Introduction. Balneotherapy is a treatment method that uses natural factors with recognized therapeutic properties based on chemical, mechanical and thermal effects on the organism. Carbonated mineral water baths are a treatment method used in the prevention and therapy of cardiovascular diseases in some Romanian spa resorts such as Băile Tuşnad. Carbon dioxide baths have an old history and are considered effective in the treatment of peripheral vascular disorders [1].

Carbonated mineral waters are the result of filtration of depth waters through volcanic soils that contain carbon dioxide. The carbon dioxide obtained in this way favors the dissolution of other elements present in the soil layers through which water passes: calcium, magnesium, sodium, iron, chloride, bromide, so that finally, carbonated mineral waters will have a complex composition. The therapeutic effects of carbonated mineral water baths are based on the action of carbon dioxide and less on the pharmacodynamic action of mineral salts in the composition of mineral water [2].

According to some authors, carbon dioxide baths might represent an effective therapeutic method in the rehabilitation of coronary heart disease, myocardial infarction and stroke, as well as in the treatment of chronic venous insufficiency, inflammatory diseases and functional disorders $[3,4]$. 
The literature reports many indications of carbonated mineral water baths, with clear evidence from controlled trials only for a small number of these, mainly for chronic circulatory disorders based on atherosclerotic disease such as peripheral arterial occlusive disease, trophic ulcerations, microangiopathy of various origins, and mild arterial hypertension [5].

The three main effects of immersion in carbonated water are a reduction of central temperature, an increase in cutaneous blood flow, and an increase in the thermal sensation score [6]. In a complete bath, 10 to more than $80 \mathrm{ml} / \mathrm{min} / \mathrm{m}^{2}$ carbon dioxide are absorbed through the skin surface, with a mean of 30 $\mathrm{ml} / \mathrm{min} / \mathrm{m}^{2}$ carbon dioxide (equivalent to 1.8 $4.5 \mathrm{l} / \mathrm{h}$ ), depending on concentration, the measurement method and temperature. This corresponds to $10 \%-12 \%$ of the amount of carbon dioxide to which the body is exposed during this period. Consequently, carbon dioxide absorption by the body is 100 times higher than water absorption [7]. Carbon dioxide that is additionally taken by the body from the bath is rapidly exhaled, so that no obvious change in blood carbon dioxide concentration occurs.

Carbonated mineral water baths have temperatures below $34^{\circ} \mathrm{C}$, below the point of thermal indifference of the water, being hypothermic baths.

According to clinical observations, the effects of carbonated baths on human subjects are: bradycardia, slight changes in blood pressure, an increase in the temperature of the exposed skin [8].

A study performed in a group of 20,000 patients $(80 \%$ with intermittent claudication stage II) treated with thermal gas (99.5\% CO2), a therapy provided by means of thermal water or dry gas for 3 weeks in a treatment center in Royat (Auvergne), demonstrated the local vasodilator effects of $\mathrm{CO} 2$, as well as an increase in the walking distance and an improvement in post-treatment ankle blood pressure compared to a control group [9].

A recent study (Italy) was conducted to investigate the specific effects of successive CO2 baths on the release of plasma free radicals, being the first study regarding oxidative status markers and carbonated mineral water therapy. The aim of this study was to assess the release of free oxygen radicals in patients with chronic arterial occlusive disease treated with carbonated mineral water. A reduction of oxidative stress was observed after 2 weeks of carbonated mineral water treatment in patients with chronic arterial occlusive disease stage II. Based on the data of this study, it is considered that an increase in the neutralization of free radicals is beneficial in arterial occlusive disease and that this neutralization can reduce systemic and local inflammatory response after ischemia-reperfusion injury [10]. The neutralizing agent might be carbonated mineral water baths [11].

The skin areas immersed in the water bath turn red when human subjects bathe in $\mathrm{CO} 2$ thermal springs, and the boundaries with the non-immersed parts are clear. Although this is also seen in pure water baths at comparatively higher water temperatures, the change of color is usually observed in $\mathrm{CO} 2$ water even at relatively low temperatures $\left(<35{ }^{\circ} \mathrm{C}\right)$. Skin color changes seem to be due to capillary bed hyperemia caused by vasodilation [11]. This effect is induced by dilation of precapillary arterioles and capillaries.

Following studies conducted in Vatra Dornei, hypertensive patients with atherosclerosis showed an improvement in peripheral circulation and a normalization of vascular hyperreactivity, an amelioration of collateral circulation and the claudication index assessed by radioisotopic and clinical methods, as well as a reduction of blood pressure values.

An extensive study carried out in Covasna on 1000 hypertensive patients demonstrated a hypotensive effect and capillary vasodilation assessed by capillaroscopy. In peripheral ischemic disorders, an improvement of the claudication index and oscillometric values was reported [2].

A clinical study performed in Baile Tuşnad aimed to evaluate the efficiency of natural therapeutic factors with the purpose of continuing rehabilitation treatment of post- 
stroke patients in a spa and climatic resort. Hemiparesis was the most frequent clinical sign, followed by coordination, balance and gait disorders. Patients attended rehabilitation treatment consisting of kinesiotherapy, carbonated mineral water baths for 15 minutes, aerotherapy for 30 minutes daily, massotherapy, performed daily for 16 days. The patients were clinically assessed before and at the end of treatment using the Tinetti Balance Scale, the $10-\mathrm{m}$ walking test, the Quality of Life Scale. At the end of treatment, an improvement in the walking distance, balance and quality of life of these patients was observed [12,13].

Case report. Patient N.M., aged 75, with cardiovascular risk factors, hypertension stage II B under antihypertensive treatment, grade 2 obesity, mixed dyslipidemia under statin treatment, and asymptomatic hyperuricemia, treated by drug therapy and diet, with peripheral vasodilatators and antiplatelet drugs. In 2013, he was diagnosed with lower limb peripheral ischemia syndrome stage II B Fontaine, through atherosclerotic arterial disease with bilateral lower limb arterial lesions, with $60-70 \%$ stenosis of the anterior and posterior tibial artery. He was admitted to the Institute of Cardiovascular Diseases, Cardiology I, Bucharest, for predominantly left claudication at about 100 meters, pain in the soles of the feet, subsequently in the calves, with progressive aggravation; pulse was absent in the dorsalis pedis and posterior tibial arteries, which is why balloon angioplasty of the peroneal artery and right posterior tibial artery was performed. Subsequently, he attended medical rehabilitation treatment annually, for 3 years, in Baile Tuşnad, consisting of carbonated mineral water baths for 15 minutes, aerotherapy for 30 minutes daily for stimulation of walking, massotherapy, special kinesiotherapy, Buerger exercises performed daily for 16 days. In 2016, he was admitted to the Rehabilitation Hospital Cluj-Napoca, where he received rehabilitation treatment consisting of kinesiotherapy and galvanic baths. The approval of the Ethics Committee was obtained. The patient was clinically assessed before and at the end of treatment during each hospitalization by the visual analogue scale for pain, the 10 -m walking test, adverse reactions, and arterial Doppler ultrasound at the end of hospitalization in the Rehabilitation Hospital Cluj-Napoca. There were no adverse effects during the hospitalizations. After each hospitalization in Băile Tuşnad, pain in the lower limbs improved from level 9 to level 5, the quality of gait increased with the development of claudication at 250 meters, and the walking distance also increased. Arterial Doppler ultrasound evidenced no worsening of stenosis in the left lower limb.

Discussions. Experimental analyses performed in human subjects and laboratory animals have shown that increased skin blood flow due to carbon dioxide baths results from the action of percutaneous $\mathrm{CO} 2$, determining skin vasodilation at relatively low water temperatures [11]. Carbonated mineral water baths increase skin arteriolar blood flow, which is demonstrated by the flowmeter, the vasodilator effect being directly proportional to the carbon dioxide concentration in the mineral bath. Carbon dioxide is absorbed through the skin even in the absence of gas bubbles [12]. There is vasodilation of arterioles and arteriovenous anastomoses. The temperature of the carbon dioxide bath is between $28-31^{\circ} \mathrm{C}[2,12]$. Several effects of carbonated mineral water baths have been observed. The therapeutic actions of these baths are explained by a synergism of many factors; the first one is related to immersion itself: hydrostatic pressure and the force of Archimedes which induce orthosympathetic inhibition and muscle relaxation. The second one depends on the pharmacological properties of carbon dioxide which acts directly on skin blood vessels, inducing vasodilation and the sensation of heat [11].

Conclusions. Rehabilitation treatment with natural therapeutic factors influenced the clinical and functional picture, determining a significant improvement of pain in the lower limbs, as well as an amelioration of the quality of gait in the patient with chronic arterial occlusive disease. 
It can be said that carbonated mineral water therapy has been used clinically for a long time in European countries as well as in Romanian spa resorts for cardiovascular rehabilitation, with an improvement of symptoms.

Although carbon dioxide thermal springs contain many types of mineral substances, depending on geographic location, the effects of carbonated mineral water baths seem to be attributed to high carbon dioxide concentrations. Carbon dioxide may affect cutaneous microcirculation and arterial macrocirculation. Controlled experiments in animals have demonstrated that both skin and muscle blood flow and oxygen pressure are increased during immersion [14].

Successive carbon dioxide applications can be clinically effective in patients with arterial occlusion of the lower limbs, and might also stop free radical generation, according to recent studies.

However, the desired long-lasting effects of carbonated mineral water baths can only be obtained by serial applications, which determine the final efficiency of carbon dioxide baths.

\section{References}

1. Radawski D, Dabney JM, Daugherty RM, Jr., Haddy FJ, Scott JB. Local effects of CO2 on vascular resistances and weight of the dog forelimb. Am J Physiol1972; 222:439-443

2. Munteanu Constantin. Ape minerale terapeutice, Editura Balneara Bucuresti, 2013

3. Irie $\mathrm{H}$, Tatsumi $\mathrm{T}$, Takamiya $\mathrm{M}$, Zen $\mathrm{K}$, Takahashi T, Azuma A, Tateishi K, Nomura T, Hayashi H, Nakajima N, Okigaki M, Matsubara $\mathrm{H}$. Carbon dioxide-rich water bathing enhances collateral blood flow in ischemic hindlimb via mobilization of endothelial progenitor cells and activation of NO-cGMP system. Circulation 2005;111:1523- 1529.

4. Toriyama T, Kumada Y, Matsubara T, Murata A, Ogino A, Hayashi H, Nakashima H, Takahashi H, Matsuo H, Kawahara H. Effect of artificial carbon dioxide foot bathing on critical limb ischemia (Fontaine IV) in peripheral arterial disease patients. Int Angiol 2002; 21:367-373.

5. Resch KL, Just U. [Possibilities and limits of CO2 balneotherapy]. Wien Med Wochenschr 1994;144:45-50

6. Pagourelias ED, Zorou PG, Tsaligopoulos M, Athyrous VG, Karagiannis A, Efthimiadis GK. Carbon dioxide balneotherapy and cardiovascular disease. Int $\mathrm{J}$ Biometeorol. 2011; 55:657-63.

7. Pratzel H, Aufnahme, Abgabe und Stoffwechsel von CO2 beim Kohlensäurebad. Z phys Med Blan Med Klim 1984;13:25-32.

8. Hartmann BR, Bassenge E, Hartmann M. Effects of serial percutaneous application of carbon dioxide in intermittent claudication: results of a controlled trial. Angiology 1997; 48:957-963.

9. Fabry R, Dubost JJ, Schmidt J, Body J, Schaff G, Baguet JC. Thermal treatment in arterial disease: an expensive placebo or an effective therapy? Therapie 1995; 50(2):11322.

10. Lau CS, Scott N, Shaw JW, Belch JJ. Increased activity of oxygen free radicals during reperfusion in patients with peripheral arterial disease undergoing percutaneous peripheral artery balloon angioplasty. Int Angiol 1991;10:244-246.

11. Hashimoto M, Yamamoto N. Decrease in heart rates by artificial $\mathrm{CO} 2$ hot spring bathing is inhibited by beta1-adrenoceptor blockade in anesthetized rats. J Appl Physiol 2004;96:226232

12. Dogaru Gabriela, Radulescu Alexandru. Therapeutic effects of carbonated mineral waters in cardiovascular rehabilitation. Balneo Research Journal 2015, vol. 6 (1):39-43

13. Gabriela Dogaru, Alexandra Ispas, Ioana Stănescu, Marieta Motricala, Molnár Ákos. A clinical study on the efficacy of natural therapeutic factors in Băile Tuşnad for the rehabilitation of post-stroke patients. Balneo Research Journal 2017; vol. 8 (1):5-10.

14. Komoto $Y$, Nakao $T$, Sunakawa $M$, Yorozu H. Elevation of tissue PO2 with improvement of tissue perfusion by topically applied CO2. Adv Exp Med Biol 1998; 222:637- 645. 